\title{
Conformidade em Memória de Reconhecimento: Revisão Breve e Potenciais Direções de Pesquisa****
}

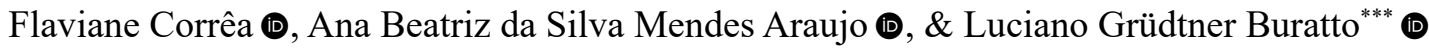 \\ Universidade de Brasília, Brasília, DF, Brasil
}

\begin{abstract}
RESUMO - Conformidade de memória é o fenômeno em que relatos de memória de uma pessoa são modificados após exposição aos relatos de outra pessoa. Estudos sobre conformidade de memória são importantes tanto no âmbito puro quanto aplicado, especialmente quando os estímulos são carregados emocionalmente. No entanto, estudos de conformidade de memória com estímulos emocionais ainda são escassos. Além disso, os poucos estudos sobre o tema apresentam resultados contraditórios. Nesta revisão, apresentamos um breve resumo de pesquisas sobre conformidade de memória em tarefas de reconhecimento e avaliamos o papel moderador da emocionalidade na conformidade. Sugerimos como possível direção de pesquisas futuras a avaliação de duas variáveis, intervalo de retenção e distintividade dos estímulos, que podem ser cruciais para elucidar as inconsistências empíricas.
\end{abstract}

PALAVRAS-CHAVE: memória de reconhecimento, conformidade de memória, emoção, intervalo de retenção, distintividade

\section{Memory Conformity in Recognition: Brief Review and Research Directions}

\begin{abstract}
Memory conformity refers to the phenomenon in which memory reports from one person are modified by memory reports from another person. The study of memory conformity is important both in basic and applied settings, especially when the stimuli are emotionally-laden. Research on memory conformity for emotional stimuli, however, is surprisingly scarce. Here we briefly review research on memory conformity in recognition tasks and assess the modulatory role of emotionality on conformity effects. We also propose further research based on the manipulation of two variables, retention interval and stimulus distinctiveness, that could help to reconcile the discrepant findings in the literature.
\end{abstract}

KEYWORDS: recognition memory, memory conformity, emotion, retention interval, distinctiveness

Indivíduos frequentemente compartilham entre si informações sobre um evento previamente testemunhado. Ao compartilhar suas lembranças, eles podem modificar detalhes do evento original. Dessa forma, relatos de memória podem ser uma mistura de informações verídicas com informações errôneas sugeridas pelos outros (Wright et al., 2009). Esse fenômeno de mudança de relatos de memória via interação social é conhecido como conformidade de memória ou contágio social da memória (Hirst \& Echterhoff, 2012; Roediger et al., 2001; Wright et al., 2000).
Estudos sobre conformidade de memória são importantes em termos teóricos e práticos. Em termos teóricos, investigar a conformidade de memória pode ajudar a entender aspectos centrais do funcionamento da memória. A memória humana é suscetível a erros (Schacter \& Slotnick, 2004) e uma parte deles são induzidos por interações sociais (Hirst \& Echterhoff, 2012). A suscetibilidade a erros no sistema de memória humano sugere que erros podem ser adaptativos (Schacter et al., 2011). Nessa visão, erros seriam o reflexo visível de um sistema capaz de modificar registros já estabilizados

\footnotetext{
* Agradecemos a Ana Paula Sousa, Giulia Veiga de Leite Ribeiro Melo, Nadia Alcoragi, Tatiana Ferreira de Araujo Litvin e Vitória Machado Dias pela colaboração de na realização do levantamento bibliográfico e pelas discussões sobre o material. Apoio: Capes (Coordenação de Aperfeiçoamento de Pessoal de Nível Superior).

** Este trabalho é baseado na dissertação de mestrado da primeira autora.

*** E-mail: lburatto@unb.br

n Submetido: 20/06/2018; Aceito: 26/06/2019.
} 
(Nader \& Hardt, 2009), adicionando novas informações que sejam relevantes para objetivos futuros. Ainda segundo essa visão, modificar memórias consolidadas via interação social seria uma característica intrínseca desse sistema, não um efeito colateral indesejável. Dessa forma, entender os fatores que resultam em erros de memória pode lançar luz sobre aspectos básicos sobre o funcionamento da memória humana. Em termos práticos, é importante entender como as interações sociais alteram relatos de memória. No contexto forense, por exemplo, testemunhas de crimes muitas vezes conversam entre si sobre o evento testemunhado (Paterson \& Kemp, 2006a; Skagerberg \& Wright, 2008b). Esse tipo de interação entre testemunhas exerce grande influência sobre seus relatos posteriores, o que pode alterar o curso de um julgamento (Paterson \& Kemp, 2006b; Shaw et al., 1997).
O objetivo dessa revisão é apresentar um breve resumo do estado atual de pesquisas sobre conformidade de memória em tarefas de reconhecimento e discutir possibilidades de investigação em aspectos importantes, mas relativamente pouco estudados, desse fenômeno, como o papel moderador da emocionalidade dos estímulos e a influência do intervalo de retenção e da distintividade de estímulos no efeito de conformidade. Primeiramente serão descritos paradigmas experimentais comuns nessa área e os principais achados em que esses paradigmas foram empregados. Em seguida, serão revisados estudos sobre o papel da emocionalidade dos estímulos na memória e na conformidade de memória. Por fim, serão apresentadas sugestões de experimentos que poderiam lançar luz sobre os resultados contraditórios dessa literatura.

\section{CONFORMIDADE DE MEMÓRIA: PARADIGMAS, MODERADORES E BASES BIOLÓGICAS}

\section{Paradigmas Experimentais}

Diferentes paradigmas experimentais foram desenvolvidos para estudar a conformidade de memória. Em geral, um participante de um desses estudos é exposto a um evento (e.g., palavra, imagem, vídeo) e depois a uma informação sobre o evento, que pode estar correta ou incorreta (e.g., outro participante menciona fato não presente no vídeo estudado originalmente). Diz-se que houve conformidade de memória quando o relato de memória do participante sobre o evento original é modificado pela informação pós-evento.

Os relatos de memória em paradigmas de conformidade podem ser obtidos por meio de testes de recordação livre, recordação com pistas ou reconhecimento (e.g., Meade \& Roediger, 2002; Wright et al., 2008). As informações pós-evento também podem ser introduzidas de diferentes maneiras: com perguntas enviesadas, via acesso às respostas do outro participante ou via conversa direta com o outro participante (e.g., Bodner et al., 2009; Gabbert et al., 2006; Harris et al., 2017; Paterson \& Kemp, 2006b). E os relatos de memória podem ser obtidos de maneira pública, com o outro participante presente, ou de maneira privada, sem o outro participante presente (e.g., McGuire et al., 2011; Reysen, 2005, 2007).

Dois paradigmas experimentais têm sido empregados frequentemente. No primeiro, participantes memorizam uma cena (e.g., a foto de um escritório) e em seguida recordam itens da cena juntamente com um confederado (participanteator que trabalha para o pesquisador e fornece respostas programadas, verdadeira ou falsas, sem o conhecimento do participante). Durante a fase de recordação conjunta, participante e confederado recordam itens alternadamente. O confederado recorda alguns itens novos, que não faziam parte da cena original (e.g., o grampeador). $\mathrm{Na}$ fase final deste paradigma, o participante faz sozinho um teste de recordação livre e é instruído a recordar apenas itens vistos na cena original. A conformidade de memória é observada quando o participante recorda itens produzidos pelo confederado que não estavam presentes na cena original (Meade \& Roediger, 2002; Roediger et al., 2001). O efeito é observado mesmo quando o participante é avisado, antes do teste final, sobre possíveis erros do confederado e mesmo quando o confederado não está fisicamente presente durante o teste. Vários estudos que utilizaram esse paradigma relataram efeitos significativos de conformidade (Davis \& Meade, 2013; Huff et al., 2013; Huff et al., 2016; Kensinger et al., 2016, Experimento 2; McNabb \& Meade, 2014).

No segundo paradigma de conformidade de memória (Reysen, 2005; Schneider \& Watkins, 1996; Wright et al., 2005), participantes estudam uma série de estímulos e depois realizam um teste de memória de reconhecimento no qual têm acesso às respostas do confederado. $\mathrm{Na}$ fase de teste, realizada minutos ou dias após a fase de estudo, estímulos previamente estudados (itens antigos) são misturados a estímulos não estudados (itens novos). O participante deve discriminar entre esses tipos de itens, respondendo "Sim" para itens antigos (acerto) e "Não" para itens novos (rejeição correta). A habilidade do participante em discriminar corretamente itens antigos de novos é medida, fornecendo um índice de memória de reconhecimento. $\mathrm{Na}$ condição controle, o participante responde sozinho (ou antes do confederado), fornecendo uma linha da base para seu desempenho. Na condição experimental, o participante responde depois do confederado, tendo acesso às suas respostas. As respostas do confederado são programadas: algumas são corretas (acertos e rejeições corretas) e algumas são incorretas ["Sim" para itens novos (alarme falso) e "Não" para itens antigos (omissão)]. A conformidade de memória é observada quando, em relação à condição controle, as 
respostas corretas do participante aumentam após exposição às respostas corretas do confederado e diminuem após exposição às respostas incorretas do confederado. Efeitos significativos de conformidade foram observados em estudos que fizeram uso desse e de outros paradigmas similares (Allan et al., 2012; Brown \& Schaefer, 2010; Horry et al., 2012; Jaeger et al., 2012; Jaeger et al., 2012; Reysen, 2005, 2007; Schwartz \& Wright, 2012; Selmeczy \& Dobbins, 2017; Skagerberg \& Wright, 2008a; Wright et al., 2012; Wright et al., 2008; Wright et al., 2010; Wright \& Villalba, 2012; Zawadzka et al., 2016).

\section{Conformidade Normativa, Informacional e Distorções de Memória}

O estudo de conformidade social tem uma longa tradição em psicologia social (Asch, 1956; Haun et al., 2013; Sherif, 1935; Toelch \& Dolan, 2015) e sua extensão para estudos de memória de longo prazo é mais recente (Schneider \& Watkins, 1996). A distinção entre influências normativa e informacional na conformidade social foi articulada por Deutsch e Gerard (1955) em experimentos em que o julgamento se baseava em decisões perceptuais (avaliação em grupo com estímulo fisicamente presente) ou de memória de curto prazo (avaliação poucos segundos após remoção do estímulo) e mais recentemente por Wright et al. (2010) no contexto de memória de longo prazo.

Em estudos de memória, a conformidade pode ocorrer por influência normativa, por influência informacional ou por distorções de memória (Wright et al., 2010; Wright et al., 2009). Na conformidade por influência normativa, o indivíduo não muda sua crença sobre o evento original. Ele sabe que a resposta do outro está errada, mas evita discordar para evitar sanções sociais. A conformidade ocorre por motivos instrumentais. Na conformidade por influência informacional, por outro lado, o indivíduo acredita mais na acurácia da memória do outro do que na acurácia da própria memória. A memória do evento original não é necessariamente modificada. Por não se lembrar de detalhes do evento original, o indivíduo pode concordar com a resposta do outro por acreditar que o outro está correto. Crença e recordação são construtos distintos: É possível acreditar que um evento ocorreu mesmo sem se recordar dele (Scoboria et al., 2004) ou, mais raramente, recordar-se de um evento mesmo sem acreditar que ele tenha acontecido (Mazzoni et al., 2010). Na conformidade por distorção de memória, a própria memória do indivíduo é modificada, possivelmente porque o indivíduo lembra da informação fornecida pelo outro e a atribui erroneamente ao evento original (confusão em relação à fonte; Meade \& Roediger, 2002).

O papel das influências normativa e informacional na conformidade de memória foi demonstrado repetidas vezes. Infere-se que a conformidade ocorreu devido a influência normativa quando um participante segue o confederado de forma pública, mas reverte sua resposta para a resposta original de forma privada (Edelson et al., 2011). Indivíduos com maior medo de avaliação social negativa tendem a mostrar maior conformidade (Wright et al., 2010), enquanto que indivíduos com maior tendência a evitar interações sociais tendem a mostrar menor conformidade (Wright et al., 2012), padrão de resultados consistente com a hipótese de influência normativa na magnitude do efeito. A influência normativa também tende a ser maior quando as consequências de se cometer um erro de memória são baixas (Baron et al., 1996). Em termos neurobiológicos, a conformidade por influência normativa tem sido associada à maior ativação em circuitos cerebrais envolvidos na detecção de conflito de informações (córtex cingulado anterior; Deuker et al., 2013; Edelson et al., 2011) e à ação do hormônio ocitocina (Edelson et al., 2015).

A influência informacional, por outro lado, tende a ser maior quando o indivíduo acredita que o outro tem mais credibilidade (Williamson et al., 2013) ou mais confiança (Wright \& Carlucci, 2011) ou quando o desempenho na tarefa de memória está associada a consequências relevantes (Baron et al., 1996). Nesses casos, as respostas do outro são consideradas informativas, principalmente quando o participante tem pouca confiança na própria memória (Jaeger et al., 2012; Wright et al., 2005; Wright \& Villalba, 2012).

Enquanto que a distinção entre influência normativa e informacional é relativamente clara, a distinção entre estas influências e distorções de memória é mais sutil. Em estudos de monitoramento de fonte (Johnson et al., 1993), o participante deve dizer se sua resposta se refere ao evento original ou se sua resposta foi apenas sugerida pelo outro. Os resultados neste tipo de estudo indicam que os participantes atribuem erroneamente a fonte de sua memória ao evento original e não à resposta do outro (Bodner et al., 2009; Gabbert et al., 2007; Meade \& Roediger, 2002; Wright et al., 2009), sugerindo que a informação fornecida pelo outro foi internalizada pelo participante.

Estudos de neuroimagem apontam maior ativação do hipocampo em situações indicativas de distorção de memória, quando a resposta errônea dada em público é mantida na resposta privada e atribuída ao evento original (Deuker et al., 2013; Edelson et al., 2014; Edelson et al., 2011). O hipocampo é uma estrutura cerebral essencial para a criação de novas memórias episódicas (Squire, 2004) e sua ativação em situações de conformidade sugere modificação no próprio traço de memória. Outra estrutura importante para a conformidade de memória é a amígdala, região cerebral cuja ativação está associada à codificação de eventos emocionalmente significantes (Roozendaal \& McGaugh, 2011). Além de maior ativação na amígdala, há também aumento na conectividade entre hipocampo e amígdala quando participantes seguem erroneamente as respostas dos outros (Edelson et al., 2011). A atividade diferencial na amígdala é particularmente observada quando o participante acredita que a informação incorreta origina- 
se de uma fonte social (i.e., outras pessoas). Quando o participante é informado que as respostas foram produzidas por um computador, não por outras pessoas, não se observa mais nem a ativação diferencial da amígdala, nem o aumento de conectividade entre amígdala e hipocampo (Edelson et al., 2011).

Os resultados dos estudos de neuroimagem sugerem atualização do traço original de memória, não somente a mudança na crença do participante. Um dos possíveis mecanismos de atualização de traços de memória é a reconsolidação, fenômeno em que traços anteriormente consolidados podem voltar a um estado volátil e ficar sujeitos a modificações após sua recuperação (Hupbach et al., 2007; Nader \& Hardt, 2009). Reconsolidação sugere um mecanismo biológico para a conformidade de memória: A reativação de um traço de memória consolidado pode torná-lo suscetível a alterações com novas informações de uma fonte externa (Scully et al., 2017).

\section{Moderadores da Conformidade de Memória}

Diversos fatores influenciam a magnitude do efeito de conformidade. $\mathrm{O}$ efeito é maior quando o participante acredita que as respostas provêm de pessoas com maior credibilidade em termos de memória - quando o outro participante é um policial versus um eletricista em teste sobre um vídeo de crime (Williamson et al., 2013); quando o outro responde primeiro em uma conversa sobre uma cena complexa, sugerindo maior confiança do outro participante (Gabbert et al., 2006); quando o participante acredita que o outro possui melhor acuidade visual (French et al., 2011); quando o outro participante é jovem versus idoso (Davis \& Meade, 2013); quando o outro é da mesma raça que a de rostos memorizados e testados (Horry et al., 2012); e quando o participante acredita que o outro estudou os estímulos por mais tempo (Allan et al., 2012; Gabbert et al., 2007). Além disso, quanto maior o número de pessoas sugerindo uma mesma informação incorreta, maior é o efeito de conformidade (Deuker et al., 2013; Walther et al., 2002).

Nos casos citados acima, a conformidade é maior devido principalmente a fatores informacionais: os participantes tendem a seguir as respostas do outro porque acreditam que ele ou ela é fonte de repostas confiáveis. Consistente com essa hipótese, quando os participantes são informados de que as respostas do outro podem estar incorretas, o efeito de conformidade diminui (Echterhoff et al., 2005; Meade $\&$ Roediger, 2002). Fatores normativos também moderam a conformidade de memória. $\mathrm{O}$ efeito é maior quando o participante considera que o outro participante é mais poderoso do que ele (Skagerberg \& Wright, 2008a) ou quando os dois se conhecem (French et al., 2008; Hope et al., 2008). Nesses casos, há um possível custo social em discordar das respostas do outro. O efeito de conformidade é observado mesmo quando fatores normativos e informacionais são reduzidos. Por exemplo, as respostas do confederado influenciam as do participante quando ambos são estudantes, situação em que pertencem a um mesmo nível hierárquico, têm idades e níveis educacionais similares e não se conhecem. A presença física do confederado nem sempre é necessária para produzir efeitos de conformidade, basta que o participante observe as respostas do confederado (Bodner et al., 2009; Meade \& Roediger, 2002; Reysen, 2005). Talvez mais surpreendente, o efeito é observado mesmo quando a acurácia do outro é baixa (50\%: Jaeger et al., 2012; 65\%: Selmeczy \& Dobbins, 2013).

\section{EMOCIONALIDADE DOS ESTÍMULOS COMO POTENCIAL MODERADOR DA CONFORMIDADE}

\section{Memória para Estímulos Emocionais}

A emocionalidade dos estímulos é um potencial moderador da conformidade de memória. O estudo de conformidade com estímulos emocionais tem importância teórica e prática. No contexto forense, os eventos de interesse podem ser neutros (e.g., presença ou não do acusado em uma loja antes do crime) ou emocionais (e.g., presença ou não do acusado na cena de um crime presenciado pela testemunha). Entender como a emocionalidade do evento influencia sua codificação e futura recuperação é importante para guiar a aceitação ou não de testemunhos no processo legal. Estímulos emocionais podem, por exemplo, gerar uma falsa sensação de confiança na própria memória que nem sempre é acompanhada de maior acurácia (Rimmele et al., 2011; Talarico \& Rubin, 2003). Esse tipo de resultado é importante porque jurados tendem a avaliar como verdadeiros relatos produzidos por testemunham que demonstram alta confiança, mesmo quando esses relatos contêm inconsistências (Brewer \& Burke, 2002). Apesar da importância da emocionalidade dos estímulos no contexto aplicado, poucos estudos exploraram o impacto dessa variável sobre a conformidade de memória.

A emocionalidade de estímulos pode ser operacionalizada como uma combinação de valores em duas dimensões contínuas: valência, que varia entre desagradável (valência negativa), neutra e agradável (valência positiva), e alerta, que varia entre relaxante (baixo) e estimulante (alto) (Bradley et al., 1992; Bradley \& Lang, 1994). Segundo essa concepção, um estímulo pode ter valência negativa e alerta alto (e.g., imagem de criança coberta de sangue) ou valência negativa e alerta baixo (e.g., imagem de criança encolhida com expressão triste). 
Valência e alerta ativam redes neurais distintas (Kensinger \& Corkin, 2004; LaBar \& Cabeza, 2006). Enquanto o hipocampo e córtex pré-frontal aumentam sua comunicação durante a codificação de estímulos posteriormente lembrados que diferem apenas em valência, o hipocampo e amígdala aumentam sua comunicação durante a codificação de estímulos posteriormente lembrados que diferem apenas em alerta (Kensinger \& Corkin, 2004). Estímulos negativos têm sido mais estudados que positivos porque tendem a produzir efeitos de memória mais consistentes (Holland \& Kensinger, 2010; Kensinger, 2009). Um dos motivos para estímulos negativos gerarem maior consistência se deve à influência da amígdala no processo de consolidação que, em última análise, fortalece os traços de memória para estímulos negativos (LaBar \& Cabeza, 2006; Yonelinas \& Ritchey, 2015).

Estímulos emocionais são mais lembrados que estímulos não emocionais (Buchanan, 2007; Hamann, 2001; Ochsner, 2000). Parte desse benefício mnemônico se deve a fatores cognitivos associados a estímulos emocionais, não à emocionalidade em si (Bennion et al., 2013; Talmi, 2013). Estímulos emocionais chamam mais a atenção, são mais distintos e mais relacionados semanticamente entre si quando comparados a estímulos neutros. Quando esses fatores são controlados, o benefício da emoção na memória é reduzido ou desaparece (Bessette-Symons, 2018; Choi et al., 2013; Talmi et al., 2007; Talmi \& McGarry, 2012).

É possível reduzir a influência de fatores cognitivos em estudos sobre emoção e memória. O benefício da maior atenção para estímulos emocionais pode ser reduzido com instruções intencionais em tarefas de memória (i.e., informar ao participante que sua memória será testada posteriormente) e com a disponibilidade de tempo suficiente para codificação do estímulo (e.g., $2 \mathrm{~s}$ ) em condições de atenção total, não dividida (Talmi et al., 2007). O benefício da maior coesão semântica também pode ser reduzido com a construção de conjuntos de estímulos com conteúdos pareados. O pareamento pode ser feito via conteúdo dos estímulos (e.g., números similares de estímulos com animais, pessoas e objetos) ou via dados de julgamentos de similaridade (Bessette-Symons, 2018; Talmi \& McGarry, 2012). Por fim, o benefício da distintividade pode ser reduzido manipulando-se entressujeitos a emocionalidade dos estímulos. Nesse tipo de manipulação, cada grupo é exposto somente a listas puras de estímulos contendo ou apenas imagens neutras ou apenas imagens negativas. Listas puras possuem valência e/ou alerta similar. Dessa forma, diferenças de distintividade entre estímulos neutros e negativos terão impacto reduzido nos escores de memória obtidos. Listas puras também reduzem o papel da maior atenção despendida para estímulos emocionais, uma vez que se elimina a possibilidade de estímulos emocionais tomarem tempo de codificação de itens neutros. Isso ocorre quando se usam listas mistas, em que itens neutros e negativos estão misturados (manipulação intrassujeitos) (Talmi et al., 2007;
Talmi \& McGarry, 2012). Em resumo, uma série de escolhas cuidadosas no delineamento experimental pode evitar que os efeitos de emoção na memória sejam superestimados.

A ação de fatores cognitivos no efeito de emoção na memória é maior quando o intervalo entre estudo e teste é curto (e.g., minutos). Para intervalos longos (horas ou dias; Schumann et al., 2017; Sharot \& Phelps, 2004; Sharot \& Yonelinas, 2008), os efeitos da emoção na memória são atribuídos a fatores "propriamente emocionais", relacionados à maior conectividade entre hipocampo e amígdala (Ritchey et al., 2008) e à maior consolidação para estímulos emocionais que neutros (Roozendaal \& McGaugh, 2011).

Segundo a visão mais aceita, a consolidação de memórias é fortalecida para estímulos emocionais em relação a neutros porque estímulos emocionais (valência negativa e alerta alto) estimulam a liberação dos hormônios adrenalina e cortisol no sangue, que estimulam direta ou indiretamente a área basolateral da amígdala, resultando no fortalecimento dos traços de memória associados a esses estímulos (McGaugh, 2000; Roozendaal \& McGaugh, 2011). Mudanças estruturais mediadas por cortisol e por síntese de proteínas ocorrem em uma escala de tempo de horas e dias. Por isso, os fatores responsáveis pelo efeito da emoção na memória mudam de acordo com o intervalo de tempo entre codificação e recuperação: Fatores cognitivos dominam quando o intervalo é curto (e.g., minutos), enquanto que fatores "propriamente emocionais" dominam quando o intervalo é longo (e.g., dias) (Talmi, 2013).

\section{Conformidade para Estímulos Emocionais}

Estudos sobre conformidade de memória para estímulos emocionais são escassos. Mais escassos ainda são estudos sobre intervalo de retenção na conformidade para estímulos emocionais. Isso surpreende, dada a relevância dos temas e de suas tradições de pesquisa. Trabalhos iniciais sobre emocionalidade avaliaram conformidade introduzida via discussão em grupo sobre eventos autobiográficos emocionais coletivos, como a morte de uma pessoa famosa (Harris et al., 2010; Yaron-Antar \& Nachson, 2006). Mais recentemente, Harris et al. (2017) usaram confederados para induzir nos participantes conformidade sobre memórias autobiográficas relevantes. O delineamento escolhido foi similar ao de estudos de contágio social de memória (Roediger et al., 2001). O resultado mostrou que detalhes de memórias pessoais relevantes foram modificados mesmo após curta interação com confederado.

Outros estudos usaram estímulos emocionais padronizados (e.g., vídeos de acidentes) para avaliar o efeito de recordação colaborativa (Soleti et al., 2017; Wessel et al., 2015). Nesses estudos, a fase colaborativa e a fase de teste final envolveram recordação livre. No estudo de Wessel et al. (2015), no entanto, não foi incluída uma condição com eventos neutros. Dessa forma, o estudo não permitiu testar 
se estímulos emocionais são mais ou menos propensos a conformidade em comparação a estímulos neutros.

Em estudos em que a fase colaborativa envolve discussão de cada estímulo por todos os membros e respostas finais individuais, observa-se facilitação: A acurácia é maior quando há discussão do que quando não há discussão (Rajaram \& Pereira-Pasarin, 2007). Tal facilitação colaborativa foi observada em estudos com estímulos emocionais e indica maior benefício para itens emocionais (positivos e negativos) que para itens neutros (Choi et al., 2017; Kensinger et al., 2016, Experimento 1). O resultado sugere que a conformidade em um paradigma de recordação colaborativa seria mais benéfica para estímulos emocionais (mais acertos, menos rejeições corretas) que para estímulos neutros.

Em Kensinger et al. (2016, Experimento 1) e Choi et al. (2017), as respostas foram espontâneas, não manipuladas, gerando uma situação propensa a acertos. Nos estudos, havia uma correlação entre um item ser antigo ou novo e as respostas dos outros membros do grupo serem "Sim" ou "Não". Os participantes responderam muito mais "Sim" para itens antigos que para itens novos, demonstrando alta acurácia. Como estímulos emocionais tendem a ser mais lembrados, é possível que a tendência a maior conformidade para acertos seja reflexo dessa correlação, que seria maior para estímulos negativos que para estímulos neutros. A possibilidade de discussão nesse paradigma também tende a reduzir alarmes falsos, pois uma lembrança incorreta por um membro do grupo poderia ser confrontada pelos outros membros. O resultado seria uma redução nos alarmes falsos e um aumento na acurácia (error pruning; Rajaram \& Pereira-Pasarin, 2010). Portanto, uma redução nos alarmes falsos em Kensinger et al. (2016, Experimento 1) e Choi et al. (2017) seria indicativa de maior conformidade, não de menor conformidade como no paradigma experimental em que não há discussão (Wright et al., 2005). Não houve, porém, diferença na conformidade para alarmes falsos em função da emocionalidade nesses dois estudos. De maneira tentativa, os estudos de Kensinger et al. (2016, Experimento 1) e Choi et al. (2017) parecem sugerir que itens emocionais são mais sujeitos a conformidade que itens neutros.

Kensinger et al. (2016, Experimento 2) também avaliaram conformidade usando o paradigma de contágio social (Roediger et al., 2001). A conformidade foi induzida com respostas manipuladas via confederado, eliminando as limitações do Experimento 1, como a correlação entre respostas corretas e discussão de respostas e a menor propensão a erros observada naquele experimento. No Experimento 2, também houve facilitação colaborativa (maior acurácia para estímulos emocionais que neutros), mas a conformidade foi menor para estímulos emocionais. Menos alarmes falsos foram produzidos na fase de teste para itens negativos introduzidos pelo confederado durante a fase colaborativa. Em conjunto, os achados de Kensinger et al. (2016, Experimentos 1 e 2), e Choi et al. (2017) indicam que estímulos emocionais podem ser mais ou menos sujeitos a conformidade de memória em função do modo como a informação social é introduzida.

Uma hipótese razoável é a de que estímulos emocionais deveriam ser sempre menos suscetíveis a conformidade que estímulos neutros em estudos com o paradigma de conformidade sem discussão (Wright et al., 2005). Há dois motivos para tal hipótese. Primeiro, estímulos antigos negativos são menos esquecidos que estímulos antigos neutros (e.g., Choi et al., 2013), o que aumentaria a resistência à introdução de erros do tipo omissão (miss rate) por um confederado (Wright et al., 2005). Segundo, estímulos novos negativos são mais distintos que estímulos novos neutros (Schmidt, 1991), fornecendo uma estratégia metamnemônica para rejeitar alarmes falsos induzidos por um confederado (Walther et al., 2002). Dessa forma, tanto respostas para estímulos antigos quanto para estímulos novos deveriam ser menos sujeitas a conformidade quando o estímulo possui natureza emocional.

Contudo, dois outros estudos mostram como ainda é incerto o papel moderador da emoção na conformidade de memória. A favor desse papel moderador, Brown e Schaefer (2010) avaliaram conformidade induzida por confederado em teste de reconhecimento e encontraram (a) conformidade significativamente menor para estímulos positivos que para estímulos neutros e (b) tendência de menor conformidade para estímulos negativos que para estímulos neutros. Em contraste aos resultados de Brown e Schaefer (2010), Wright et al. (2012) testaram conformidade com respostas espontâneas de dois participantes que alternavam suas respostas em uma tarefa de reconhecimento e não observaram moderação do efeito de conformidade em função da emocionalidade dos estímulos: O efeito foi similar para estímulos positivos, negativos e neutros.

Os resultados de Brown e Schaefer (2010) e de Wright et al. (2012) foram em direções opostas ao esperado. Brown e Schaefer (2010) manipularam emocionalidade com listas puras (estímulos emocionais ou neutros) e sem intervalo de retenção, enquanto Wright et al. (2012) manipularam emocionalidade com listas mistas (estímulos emocionais $e$ neutros) e com um intervalo de retenção de 2 dias. Os tipos de lista e intervalo adotados deveriam reduzir o efeito de emoção na memória em Brown e Schaefer (2010) e aumentar esse efeito em Wright et al. (2012). O que se observou, no entanto, foi o oposto (mais detalhes na seção Direções Futuras).

Embora os quatro estudos revisados tenham apresentado efeitos significativos de conformidade e de emoção na memória (Brown \& Schaefer, 2010; Choi et al., 2017; Kensinger et al., 2016; Wright et al., 2012), a interação entre conformidade e emocionalidade não se mostrou consistente, apontando ora para menor, ora para maior, ora para nenhuma diferença na conformidade entre estímulos emocionais e neutros. 


\section{Direções Futuras}

As metodologias e resultados contraditórios dificultam conclusões sobre o papel moderador de emoção na conformidade de memória. Contudo, as diferenças entre os estudos também sugerem uma estratégia de investigação baseada na manipulação fatorial de duas variáveis-chave: o intervalo de retenção e a distintividade dos estímulos. Além de importantes moduladores do efeito de emoção na memória, essas variáveis foram tratadas de forma diferente nos estudos revisados, dificultando sua comparabilidade.

Um estudo futuro, por exemplo, poderia fixar um paradigma de conformidade, como o usado por Brown e Schaefer (2010) em teste reconhecimento, com presença de confederado e sem possibilidade de discussão de respostas. Nesse delineamento, o intervalo de retenção poderia variar entre curto (e.g., 5 minutos) e longo (e.g., 2 dias) e a distintividade poderia variar entre baixa (e.g., emocionalidade manipulada entressujeitos com listas puras) e alta (e.g., emocionalidade manipulada intrassujeitos com listas mistas).

O intervalo curto com listas puras replicaria o delineamento de Brown e Schaefer (2010), que encontraram menor conformidade para estímulos emocionais. O intervalo curto naquele estudo pode ter reduzido o efeito moderador de emoção na conformidade. Efeitos maiores de conformidade são observados para intervalos mais longos (Schwartz \& Wright, 2012) e, de maneira relacionada, para estímulos associados a traços de memória mais fracos (Wright et al., 2005). Intervalos mais longos são também associados a maiores efeitos de emoção na memória (Schumann et al., 2017; Sharot \& Yonelinas, 2008). Assim, para intervalos mais longos seria esperado um aumento no efeito moderador da emocionalidade sobre a conformidade. Em outras palavras, a interação emoção $\times$ resposta do confederado deveria ser maior após intervalo longo (o que representaria uma nova condição experimental) do que após intervalo curto (o que representaria uma replicação de Brown \& Schaefer, 2010).

No outro extremo, o intervalo longo com listas mistas replicaria o delineamento de Wright et al. (2012). A manipulação de emoção intrassujeitos aumenta a distintividade de estímulos emocionais em relação aos neutros. Com isso, o efeito de emoção na memória deveria aumentar (Talmi et al., 2007). A combinação de longo intervalo de retenção e uso de mistas listas forneceria condições ótimas para a detecção de efeitos de emoção na memória (Talmi, 2013) e, consequentemente, para a detecção de moderação da conformidade pela emocionalidade dos estímulos. Apesar disso, Wright et al. (2012) não encontraram diferença de conformidade entre estímulos emocionais e neutros.

Outras diferenças metodológicas entre Brown e Schaefer (2010) e Wright et al. (2012) poderiam contribuir para os resultados. Por exemplo, no primeiro as respostas foram dadas por um confederado com acurácia definida, enquanto no segundo respostas corretas e incorretas foram dadas espontaneamente. Intervalo de retenção e distintividade, no entanto, são manipulações cujos efeitos na memória têm sido mais estudados e, por isso, seriam sugestões de manipulações iniciais para lançar luz sobre esses achados contraditórios.

No experimento proposto, as outras duas condições - intervalo longo/lista pura e intervalo curto/lista mista - ajudariam a elucidar inconsistências na literatura. Por exemplo, é possível que os estímulos neutros não esquecidos após 2 dias demonstrem maior resistência a conformidade devido ao mesmo processo de consolidação que também fortalece estímulos emocionais (McGaugh, 2000). Nesse caso, embora menos numerosos que estímulos emocionais, estímulos neutros mostrariam conformidade similar a estímulos emocionais tanto no intervalo longo/lista mista, replicando Wright et al. (2012), quanto no intervalo longo/ lista pura (nova condição experimental). Se por outro lado, a interação emoção $\times$ resposta do confederado depender do tipo de lista (mista vs. pura) no intervalo longo, o resultado indicaria que memórias consolidadas são diferencialmente suscetíveis a conformidade dependendo da distintividade dos estímulos, não necessariamente da sua emocionalidade.

Também é possível que intervalo de retenção (intervalo longo vs. curto) não afete diferencialmente a conformidade para estímulos emocionais e neutros. Tal resultado indicaria que consolidação (McGaugh, 2000; Roozendaal $\&$ McGaugh, 2011) por si só não é suficiente para explicar resultados anteriores. De fato, Brown e Schaefer (2010) testaram participantes imediatamente após a fase de estudo, situação em que a consolidação é improvável, e observaram menor conformidade para estímulos emocionais. Kensinger et al. (2016, Experimento 2), por outro lado, testaram participantes após 2 dias, quando a consolidação é provável, e também observaram menor conformidade para estímulos emocionais. Os resultados haviam sugerido que consolidação não seria um fator crucial na interação emoção $\times$ resposta do confederado. Contudo, diferenças metodológicas dificultavam a interpretação. No estudo sugerido, intervalo de retenção seria manipulado num mesmo experimento, mantendo-se fixas outras variáveis e facilitando a interpretabilidade dos resultados.

O papel da consolidação na conformidade para estímulos emocionais pode inclusive ser o inverso do descrito anteriormente, tendo em vista os achados de que estímulos consolidados, quando reativados, podem ser até mais sujeitos a influências externas (Scully et al., 2017). Mais pesquisas com intervalo de retenção são, portanto, necessárias para esclarecer o papel da consolidação na conformidade de memória para estímulos emocionais.

\section{Conclusões}

Conformidade de memória é um fenômeno importante em contexto básicos e aplicados, principalmente quando 
envolve estímulos de natureza emocional. No entanto, ainda há poucos estudos de conformidade com estímulos emocionais, e os resultados destes estudos são contraditórios. Nessa revisão, apresentamos um resumo desses achados e sugerimos como possível direção de pesquisa a avaliação sistemática de duas variáveis, intervalo de retenção e distintividade dos estímulos, o que poderia ajudar a elucidar as inconsistências empíricas.

\section{REFERÊNCIAS}

Allan, K., Midjord, J. P., Martin, D., \& Gabbert, F. (2012). Memory Conformity and the Perceived Accuracy of Self versus Other. Memory \& Cognition, 40(2), 280-286. https://doi.org/10.3758/ s13421-011-0141-9

Asch, S. E. (1956). Studies of Independence and Conformity: I. A Minority of One against a Unanimous majority. Psychological Monographs: General and Applied, 70(9), 1-70. https://doi. org/10.1037/h0093718

Baron, R. S., Vandello, J. A., \& Brunsman, B. (1996). The Forgotten Variable in Conformity Research: Impact of Task Importance on Social Influence. Journal of Personality and Social Psychology, 71, 915-927. https://doi.org/10.1037/00223514.71.5.915

Bennion, K. A., Ford, J. H., Murray, B. D., \& Kensinger, E. A. (2013). Oversimplification in the Study of Emotional Memory. Journal of the International Neuropsychological Society, 19, 953-961. https://doi.org/10.1017/S1355617713000945

Bessette-Symons, B. A. (2018). The Robustness of False Memory for Emotional Pictures. Memory, 26, 171-188. https://doi.org $/ 10.1080 / 09658211.2017 .1339091$

Bodner, G. E., Musch, E., \& Azad, T. (2009). Reevaluating the Potency of the Memory Conformity Effect. Memory \& Cognition, 37, 1069-1076. https://doi.org/10.3758/ MC.37.8.1069

Bradley, M. M., Greenwald, M. K., Petry, M. C., \& Lang, P. J. (1992) Remembering Pictures: Pleasure and Arousal in Memory. Journal of Experimental Psychology: Learning, Memory, and Cognition, 18, 379-390. https://doi.org/10.1037/02787393.18.2.379

Bradley, M. M., \& Lang, P. J. (1994). Measuring Emotion: The SelfAssessment Manikin and the Semantic Differential. Journal of Behavior Therapy and Experimental Psychiatry, 25, 49-59. https://doi.org/10.1016/0005-7916(94)90063-9

Brewer, N., \& Burke, A. (2002). Effects of Testimonial Inconsistencies and Eyewitness Confidence on Mock-Juror Judgments. Law and Human Behavior, 26, 353-364. https:// doi.org/10.1023/A:1015380522722

Brown, C., \& Schaefer, A. (2010). The Effects of Conformity on Recognition Judgments for Emotional Stimuli. Acta Psychologica, 133, 38-44. https://doi.org/10.1016/j. actpsy.2009.08.004

Buchanan, T. W. (2007). Retrieval of Emotional Memories. Psychological Bulletin, 133, 761-779. https://doi. org/10.1037/0033-2909.133.5.761

Choi, H. Y., Kensinger, E. A., \& Rajaram, S. (2013). Emotional Content Enhances True but not False Memory for Categorized Stimuli. Memory \& Cognition, 41(3), 403-415. https://doi. org/10.3758/s13421-012-0269-2

Choi, H. Y., Kensinger, E. A., \& Rajaram, S. (2017). Mnemonic Transmission, Social Contagion, and Emergence of Collective Memory: Influence of Emotional Valence, Group Structure, and Information Distribution. Journal of Experimental Psychology: General, 146(9), 1247-1265. https://doi.org/10.1037/ xge0000327

Davis, S. D., \& Meade, M. L. (2013). Both Young and Older Adults Discount Suggestions from Older Adults on a Social Memory Test. Psychonomic Bulletin \& Review, 20, 760-765. https://doi. org/10.3758/s13423-013-0392-5
Deuker, L., Muller, A. R., Montag, C., Markett, S., Reuter, M., Fell, J., Trautner, P., \& Axmacher, N. (2013). Playing Nice: A MultiMethodological Study on the Effects of Social Conformity on Memory. Frontiers in Human Neuroscience, 7, 79. https://doi. org/10.3389/fnhum.2013.00079

Deutsch, M., \& Gerard, H. B. (1955). A Study of Normative and Informational Social Influences upon Individual Judgment. Journal of Abnormal Psychology, 51, 629-636. https://doi. $\operatorname{org} / 10.1037 / \mathrm{h} 0046408$

Echterhoff, G., Hirst, W., \& Hussy, W. (2005). How Eyewitnesses Resist Misinformation: Social Postwarnings and the Monitoring of Memory Characteristics. Memory \& Cognition, 33, 770-782. https://doi.org/10.3758/BF03193073

Edelson, M. G., Dudai, Y., Dolan, R. J., \& Sharot, T. (2014). Brain substrates of recovery from misleading influence. Journal of Neuroscience, 34, 7744-7753. https://doi.org/10.1523/ JNEUROSCI.4720-13.2014

Edelson, M. G., Sharot, T., Dolan, R. J., \& Dudai, Y. (2011). Following the Crowd: Brain Substrates of Long-Term Memory Conformity. Science, 333, 108-111. https://doi.org/10.1126/ science. 1203557

Edelson, M. G., Shemesh, M., Weizman, A., Yariv, S., Sharot, T., \& Dudai, Y. (2015). Opposing Effects of Oxytocin on Overt Compliance and Lasting Changes to Memory. Neuropsychopharmacology, 40, 966-973. https://doi. org/10.1038/npp.2014.273

French, L., Garry, M., \& Mori, K. (2008). You Say Tomato? Collaborative Remembering Leads to More False Memories for Intimate Couples than for Strangers. Memory, 16, 262-273. https://doi.org/10.1080/09658210701801491

French, L., Garry, M., \& Mori, K. (2011). Relative - not Absolute - Judgments of Credibility Affect Susceptibility to Misinformation Conveyed During Discussion. Acta Psychologica, 136, 119-128. https://doi.org/10.1016/j. actpsy.2010.10.009

Gabbert, F., Memon, A., \& Wright, D. B. (2006). Memory Conformity: Disentangling the Steps toward Influence During a Discussion. Psychonomic Bulletin \& Review, 13, 480-485. https://doi.org/10.3758/BF03193873

Gabbert, F., Memon, A., \& Wright, D. B. (2007). I Saw It for Longer than You: The Relationship between Perceived Encoding Duration and Memory Conformity. Acta Psychologica, 124, 319-331. https://doi.org/10.1016/j. actpsy.2006.03.009

Hamann, S. (2001). Cognitive and Neural Mechanisms of Emotional Memory. Trends in Cognitive Sciences, 5, 394-400. https://doi. org/10.1016/S1364-6613(00)01707-1

Harris, C. B., Barnier, A. J., Sutton, J., \& Keil, P. G. (2010). How did you feel when "The Crocodile Hunter" died? Voicing and silencing in conversation influences memory for an autobiographical event. Memory, 18, 185-197. https://doi. org/10.1080/09658210903153915

Harris, C. B., Barnier, A. J., Sutton, J., \& Khan, T. (2017). Social Contagion of Autobiographical Memories. Journal of Applied Research in Memory and Cognition, 6, 319-327. https://doi. org/10.1016/j.jarmac.2017.07.006

Haun, D. B., van Leeuwen, E. J., \& Edelson, M. G. (2013). Majority Influence in Children and other Animals. Developmental 
Cognitive Neuroscience, 3, 61-71. https://doi.org/10.1016/j. dcn.2012.09.003

Hirst, W., \& Echterhoff, G. (2012). Remembering in Conversations: The Social Sharing and Reshaping of Memories. Annual Review of Psychology, 63, 55-79. https://doi.org/10.1146/ annurev-psych-120710-100340

Holland, A. C., \& Kensinger, E. A. (2010). Emotion and Autobiographical Memory. Physics of Life Reviews, 7, 88-131. https://doi.org/10.1016/j.plrev.2010.01.006

Hope, L., Ost, J., Gabbert, F., Healey, S., \& Lenton, E. (2008). "With a Little Help from My Friends...": The Role of CoWitness Relationship in Susceptibility to Misinformation. Acta Pscyhologica, 127, 476-484. https://doi.org/10.1016/j. actpsy.2007.08.010

Horry, R., Palmer, M. A., Sexton, M. L., \& Brewer, N. (2012). Memory Conformity for Confidently Recognized Items: The Power of Social Influence on Memory Reports. Journal of Experimental Social Psychology, 48, 783-786. https://doi. org/10.1016/j.jesp.2011.12.010

Huff, M. J., Davis, S. D., \& Meade, M. L. (2013). The Effects of Initial Testing on False Recall and False Recognition in the Social Contagion of Memory Paradigm. Memory \& Cognition, 41(6), 820-831. https://doi.org/10.3758/s13421-013-0299-4

Huff, M. J., Weinsheimer, C. C., \& Bodner, G. E. (2016). Reducing the Misinformation Effect Through Initial Testing: Take Two Tests and Recall Me in the Morning? Applied Cognitive Psychology, 30, 61-69. https://doi.org/10.1002/acp.3167

Hupbach, A., Gomez, R., Hardt, O., \& Nadel, L. (2007). Reconsolidation of Episodic Memories: A Subtle Reminder Triggers Integration of New Information. Learning \& Memory, 14, 47-53. https://doi.org/10.1101/lm.365707

Jaeger, A., Cox, J. C., \& Dobbins, I. G. (2012). Recognition Confidence Under Violated and Confirmed Memory Expectations. Journal of Experimental Psychology: General, 141, 282-301. https://doi.org/10.1037/a0025687

Jaeger, A., Lauris, P., Selmeczy, D., \& Dobbins, I. G. (2012). The Costs and Benefits of Memory Conformity. Memory \& Cognition, 40, 101-112. https://doi.org/10.3758/s13421-0110130-z

Johnson, M. K., Hashtroudi, S., \& Lindsay, D. S. (1993). Source Monitoring. Psychological Bulletin, 114, 3-28.

Kensinger, E. A. (2009). Remembering the Details: Effects of Emotion. Emotion Review, 1, 99-113. https://doi. org/10.1177/1754073908100432

Kensinger, E. A., Choi, H. Y., Murray, B. D., \& Rajaram, S. (2016). How Social Interactions Affect Emotional Memory Accuracy: Evidence from Collaborative Retrieval and Social Contagion Paradigms. Memory \& Cognition, 44(5), 706-716. https://doi. org/10.3758/s13421-016-0597-8

Kensinger, E. A., \& Corkin, S. (2004). Two Routes to Emotional Memory: Distinct Neural Processes for Valence and Arousal. Proceedings of the National Academy of Sciences of the United States of America, 101, 3310-3315. https://doi.org/10.1073/ pnas.0306408101

LaBar, K. S., \& Cabeza, R. (2006). Cognitive Neuroscience of Emotional Memory. Nature Reviews Neuroscience, 7, 54-64. https://doi.org/10.1038/nrn1825

Mazzoni, G., Scoboria, A., \& Harvey, L. (2010). Nonbelieved Memories. Psychological Science, 21, 1334-1340. https://doi/ org/10.1177/0956797610379865

McGaugh, J. L. (2000). Memory: ACentury of Consolidation. Science, 287, 248-251. https://doi.org/10.1126/science.287.5451.248

McGuire, K., London, K., \& Wright, D. B. (2011). Peer Influence on Event Reports among Adolescents and Young Adults. Memory, 19, 674-683. https://doi.org/10.1080/09658211.2011.602086

McNabb, J. C., \& Meade, M. L. (2014). Correcting Socially Introduced False Memories: The Effect of Re-Study. Journal of Applied Research in Memory and Cognition, 3, 287-292. https://doi.org/10.1016/j.jarmac.2014.05.007

Meade, M. L., \& Roediger, H. L. (2002). Explorations in the Social Contagion of Memory. Memory \& Cognition, 30(7), 995-1009. https://doi.org/10.3758/BF03194318

Nader, K., \& Hardt, O. (2009). A Single Standard for Memory: The Case for Reconsolidation. Nature Reviews Neuroscience, 10, 224-234. https://doi.org/10.1038/nrn2590

Ochsner, K. N. (2000). Are Affective Events Richly Recollected or Simply Familiar? The Experience and Process of Recognizing Feelings Past. Journal of Experimental Psychology: General, 129(2), 242-261. https://doi.org/10.1037/0096-3445.129.2.242

Paterson, H. M., \& Kemp, R. I. (2006a). Co-Witnesses Talk: A Survey of Eyewitness Discussion. Psychology Crime \& Law, 12, 181-191. https://doi.org/10.1080/10683160512331316334

Paterson, H. M., \& Kemp, R. I. (2006b). Comparing Methods of Encountering Post-Event Information: The Power of CoWitness Suggestion. Applied Cognitive Psychology, 20(8), 1083-1099. https://doi.org/10.1002/acp.1261

Rajaram, S., \& Pereira-Pasarin, L. P. (2007). Collaboration Can Improve Individual Recognition Memory: Evidence from Immediate and Delayed Tests. Psychonomic Bulletin \& Review, 14, 95-100. https://doi.org/10.3758/BF03194034

Rajaram, S., \& Pereira-Pasarin, L. P. (2010). Collaborative Memory: Cognitive Research and Theory. Perspectives on Psychological Science, 5, 649-663. https://doi.irg/10.1177/1745691610388763

Reysen, M. B. (2005). The Effects of Conformity on Recognition Judgements. Memory, 13, 87-94. https://doi. org $/ 10.1080 / 09658210344000602$

Reysen, M. B. (2007). The Effects of Social Pressure on False Memories. Memory \& Cognition, 35, 59-65. https://doi. org/10.3758/BF03195942

Rimmele, U., Davachi, L., Petrov, R., Dougal, S., \& Phelps, E. A. (2011). Emotion Enhances the Subjective Feeling of Remembering, Despite Lower Accuracy for Contextual Details. Emotion, 11(3), 553-562. https://doi.org/10.1037/a0024246

Ritchey, M., Dolcos, F., \& Cabeza, R. (2008). Role of Amygdala Connectivity in the Persistence of Emotional Memories Over Time: An Event-Related fMRI Investigation. Cerebral Cortex, 18, 2494-2504. https://doi.org/d10.1093/cercor/bhm262

Roediger, H. L., Meade, M. L., \& Bergman, E. T. (2001). Social Contagion of Memory. Psychonomic Bulletin \& Review, 8(2), 365-371. https://doi.org/10.3758/BF03196174

Roozendaal, B., \& McGaugh, J. L. (2011). Memory Modulation. Behavioral Neuroscience, 125, 797-824. https://doi. org/10.1037/a0026187

Schacter, D. L., Guerin, S. A., \& St. Jacques, P. L. (2011). Memory Distortion: An Adaptive Perspective. Trends in Cognitive Sciences, 15, 467-474. https://doi.org/10.1016/j. tics.2011.08.004

Schacter, D. L., \& Slotnick, S. D. (2004). The Cognitive Neuroscience of Memory Distortion. Neuron, 44, 149-160. https://doi.org/10.1016/j.neuron.2004.08.017

Schmidt, S. R. (1991). Can We Have a Distinctive Theory of Memory? Memory \& Cognition, 19, 523-542. https://doi. org/10.3758/bf03197149

Schneider, D. M., \& Watkins, M. J. (1996). Response Conformity in Recognition Testing. Psychonomic Bulletin \& Review, 3, 481-485. https://doi.org/10.3758/BF03214550

Schumann, D., Bayer, J., Talmi, D., \& Sommer, T. (2017). Dissociation of Immediate and Delayed Effects of Emotional Arousal on Episodic Memory. Neurobiology of Learning and Memory, 148, 11-19. https://doi.org/10.1016/j.nlm.2017.12.007

Schwartz, S. L., \& Wright, D. B. (2012). Memory Conformity for New and Old Items with Immediate and Delayed Testing. Applied Cognitive Psychology, 26, 508-515. https://doi. org/10.1002/acp. 2820 
Scoboria, A., Mazzoni, G., Kirsch, I., \& Relyea, M. (2004). Plausibility and Belief in Autobiographical Memory. Applied Cognitive Psychology, 18, 791-807. https://doi.org/10.1002/ acp. 1062

Scully, I. D., Napper, L. E., \& Hupbach, A. (2017). Does Reactivation Trigger Episodic Memory Change? A MetaAnalysis. Neurobiology of Learning and Memory, 142, 99-107. https://doi.org/10.1016/j.nlm.2016.12.012

Selmeczy, D., \& Dobbins, I. G. (2013). Metacognitive Awareness and Adaptive Recognition Biases. Journal of Experimental Psychology: Learning, Memory, and Cognition, 39, 678-690. https://doi.org/10.1037/a0029469

Selmeczy, D., \& Dobbins, I. G. (2017). Ignoring Memory Hints: The Stubborn Influence of Environmental Cues on Recognition Memory. Journal of Experimental Psychology: Learning, Memory, and Cognition, 43, 1448-1469. https://doi. org/10.1037/xlm0000383

Sharot, T., \& Phelps, E. A. (2004). How Arousal Modulates Memory: Disentangling the Effects of Attention and Retention. Cognitive, Affective \& Behavioral Neuroscience, 4(3), 294-306. https://doi.org/10.3758/CABN.4.3.294

Sharot, T., \& Yonelinas, A. P. (2008). Differential Time-Dependent Effects of Emotion on Recollective Experience and Memory for Contextual Information. Cognition, 106, 538-547. https:// doi.org/10.1016/j.cognition.2007.03.002

Shaw, J. S., 3rd, Garven, S., \& Wood, J. M. (1997). Co-Witness Information Can Have Immediate Effects on Eyewitness Memory Reports. Law and Human Behavior, 21, 503-523. https://doi.org/10.1023/A:1024875723399

Sherif, M. (1935). A Study of Some Social Factors in Perception. Archives of Psychology, 27(187), 1-60.

Skagerberg, E. M., \& Wright, D. B. (2008a). Manipulating power Can Affect Memory Conformity. Applied Cognitive Psychology, 22, 207-216. https://doi.org/10.1002/acp.1353

Skagerberg, E. M., \& Wright, D. B. (2008b). The Prevalence of Co-Witnesses and Co-Witness Discussions in Real Eyewitnesses. Psychology Crime \& Law, 14, 513-521. https:// doi.org/10.1080/10683160801948980

Soleti, E., Wright, D. B., \& Curci, A. (2017). Emotional Discussions Reduce Memory Recall. Memory, 25, 697-703. https://doi.org $/ 10.1080 / 09658211.2016 .1206943$

Squire, L. R. (2004). Memory Systems of the Brain: A Brief History and Current Perspective. Neurobiology of Learning and Memory, 82, 171-177. https://doi.org/10.1016/j. nlm.2004.06.005

Talarico, J. M., \& Rubin, D. C. (2003). Confidence, Not Consistency, Characterizes Flashbulb Memories. Psychological Science, 14, 455-461. https://doi.org/10.1111/1467-9280.02453

Talmi, D. (2013). Enhanced Emotional Memory: Cognitive and Neural Mechanisms. Current Directions in Psychological Science, 22, 430-436. https://doi.org/10.1177/0963721413498893

Talmi, D., Luk, B. T. C., McGarry, L. M., \& Moscovitch, M. (2007). The Contribution of Relatedness and Distinctiveness to Emotionally-Enhanced Memory. Journal of Memory and Language, 56, 555-574. https://doi.org/10.1016/j. jm1.2007.01.002

Talmi, D., \& McGarry, L. M. (2012). Accounting for Immediate Emotional Memory Enhancement. Journal of Memory and Language, 66, 93-108. https://doi.org/10.1016/j. jml.2011.07.009

Talmi, D., Schimmack, U., Paterson, T., \& Moscovitch, M. (2007). The Role of Attention and Relatedness in Emotionally Enhanced
Memory. Emotion, 7, 89-102. https://doi.org/10.1037/15283542.7.1.89

Toelch, U., \& Dolan, R. J. (2015). Informational and Normative Influences in Conformity from a Neurocomputational Perspective. Trends in Cognitive Sciences, 19, 579-589. https:// doi.org/10.1016/j.tics.2015.07.007

Walther, E., Bless, H., Strack, F., Rackstraw, P., Wagner, D., \& Werth, L. (2002). Conformity Effects in Memory as a Function of Group Size, Dissenters and Uncertainty. Applied Cognitive Psychology, 16, 793-810. https://doi.org/10.1002/acp.828

Wessel, I., Zandstra, A. R., Hengeveld, H. M., \& Moulds, M. L. (2015). Collaborative Recall of Details of an Emotional Film. Memory, 23, 437-444. https://doi.org/10.1080/09658211.20 14.895384

Williamson, P., Weber, N., \& Robertson, M. T. (2013). The Effect of Expertise on Memory Conformity: A Test of Informational Influence. Behavioral Sciences \& the Law, 31(5), 607-623. https://doi.org/10.1002/bsl.2094

Wright, D. B., Busnello, R. H., Buratto, L. G., \& Stein, L. M. (2012). Are Valence and Social Avoidance Associated with the Memory Conformity Effect? Acta Psychologica, 141, 78-85. https://doi. org/10.1016/j.actpsy.2012.06.008

Wright, D. B., \& Carlucci, M. E. (2011). The Response Order Effect: People Believe the First Person who Remembers an Event. Psychonomic Bulletin \& Review, 18, 805-812. https:// doi.org/10.3758/s13423-011-0089-6

Wright, D. B., Gabbert, F., Memon, A., \& London, K. (2008). Changing the Criterion for Memory Conformity in Free Recall and Recognition. Memory, 16, 137-148. https://doi. org/10.1080/09658210701836174

Wright, D. B., London, K., \& Waechter, M. (2010). Social Anxiety Moderates Memory Conformity in Adolescents. Applied Cognitive Psychology, 24, 1034-1045. https://doi.org/10.1002/ acp. 1604

Wright, D. B., Mathews, S. A., \& Skagerberg, E. M. (2005). Social Recognition Memory: The Effect of Other People's Responses for Previously Seen and Unseen Items. Journal of Experimental Psychology: Applied, 11(3), 200-209. https://doi. org/10.1037/1076-898X.11.3.200

Wright, D. B., Memon, A., Skagerberg, E. M., \& Gabbert, F. (2009). When Eyewitnesses Talk. Current Directions in Psychological Science, 18, 174-178. https://doi.org/10.1111/j.14678721.2009.01631.x

Wright, D. B., Self, G., \& Justice, C. (2000). Memory Conformity: Exploring Misinformation Effects when Presented by Another Person. British Journal of Psychology, 91, 189-202. https://doi. org/10.1348/000712600161781

Wright, D. B., \& Villalba, D. K. (2012). Memory conformity Affects Inaccurate Memories More than Accurate Memories. Memory, 20, 254-265. https://doi.org/10.1080/09658211.2012.654798

Yaron-Antar,A., \& Nachson, I. (2006). Collaborative Remembering of Emotional Events: The Case of Rabin's Assassination. Memory, 14, 46-56. https://doi.org/10.1080/09658210444000502

Yonelinas, A. P., \& Ritchey, M. (2015). The Slow Forgetting of Emotional Episodic Memories: An Emotional Binding Account. Trends in Cognitive Sciences, 19, 259-267. https:// doi.org/10.1016/j.tics.2015.02.009

Zawadzka, K., Krogulska, A., Button, R., Higham, P. A., \& Hanczakowski, M. (2016). Memory, Metamemory, and Social Cues: Between Conformity and Resistance. Journal of Experimental Psychology: General, 145, 181-199. https://doi. org/10.1037/xge0000118 